\title{
Curcumin Improves the Efficacy of BMSCs in Myocardial Ischemia
} Injury in Rat

\author{
Masoumeh Zirak Javanmard ${ }^{1,{ }^{*}}$, Mehdi Rahnavard ${ }^{1}$, Hamid Soraya ${ }^{2}$ and Mojtaba Karimipour ${ }^{1}$ \\ ${ }^{1}$ Department of Anatomy, Faculty of Medicine, Urmia University of Medical Sciences, Urmia, Iran \\ ${ }^{2}$ Department of Pharmacology, Faculty of Pharmacy, Urmia University of Medical Sciences, Urmia, Iran \\ “Corresponding author: Associated Professor, Department of Anatomy, Faculty of Medicine, Urmia University of Medical Sciences, Urmia, Iran. Email: ms_zirak@yahoo.com
}

Received 2018 November 20; Revised 2019 August 04; Accepted 2019 August 17.

\begin{abstract}
Background: Myocardial infarction can be associated with irreversal myocardial ischemia and the necrosis of the affected site of the heart. Curcumin has a protective effect on Myocardial Ischemia.

Objectives: Our study aimed to combine a regenerative medicine with a traditional antioxidant effects as an adjuvant for the treatment of isoproterenol-induced MI.

Methods: This experimental study conducted on 36 adult male Wistar rats in an university-affiliated animal lab, Urmia, Iran, in 2016. Male rats were divided into six groups of six rats, including control, MI (ISO $100 \mathrm{mg} / \mathrm{kg}$ ), curcumin ( $80 \mathrm{mg} / \mathrm{kg}$ by oral gavage), Bone Marrow Mesenchymal Stem Cells (BMSCs; $4 \times$ intravenous injection), and Cur-BMSCs. The efficiency of these methods was evaluated by histopathological examination, immunohistochemistry, measurement of heart rate, heart to body weight ratio, size of infarction, and serum levels of CK-MB, LDH, MDA, and SOD.

Results: The benefit of curcumin was apparent in two recipient groups. The rats that received curcumin or were treated with BMSCs showed significant reductions in heart to body weight ratio $(\mathrm{P}<0.001)$. The elevation of serum levels of CPK, LDH, MDA, and SOD (P $<0.001)$ was done in curcumin treatment groups. Decreases in the number of apoptotic cells were significant in cur $(\mathrm{P}<0.001)$ and cur-BMSCs ( $\mathrm{P}=0.023)$ groups; histopathological injuries were recognized significantly in cur $(\mathrm{P}<0.001)$ and cur-BMSCs $(\mathrm{P}=0.012)$ groups, and infarct size was significant in both curcumin groups $(\mathrm{P}<0.001)$.

Conclusions: Curcumin pretreatment of Bone Marrow Mesenchymal Stem Cells helps in stem cells transplantation and acts as a synergist.
\end{abstract}

Keywords: Adjuvants, Bone Marrow, Creatine Kinase, Curcumin, Ischemia, Isoproterenol, Mesenchymal Stem Cells, Myocardial Ischemia, Rat, Regenerative Medicine

\section{Background}

Myocardial infarction (MI) includes the permanent and irreversible death of the part of the heart muscle due to the loss of balance between the demand and supply of blood flow and the occurrence of ischemia (1). Although much progress has been made in the treatment of MI, it remains the leading cause of death and morbidity in the world (2). It is determined by hemodynamic, biochemical, and histopathological impairment, declined endogenous antioxidants, the release of heart damage factor enzymes, and lipid peroxidation (3).

Curcumin is a natural antioxidant that attracts the attention of researchers with its anti-inflammatory, antiapoptosis, and anti-oxidant activities. Curcumin can improve the area of infarction by changing biochemical biomarkers, reducing inflammation and fibrosis, and in- creasing angiogenesis (4).

Bone Marrow Mesenchymal Stem Cells (BMSCs) may play their protective role by releasing growth factors, cytockines and lipid microvesicles in intracellular space. They can release these vesicles even in hypoxic conditions to repair the ischemic area and promote heart function (5). BMSCs were first used for the treatment of isoproterenolinduced MI two years ago (6). Some research has examined the effect of MSCs combined with growth factors (7), nanoparticles (8), and silk fibroin- hyaluronic acid (9).

The high-dose consumption of Isoproterenol induces severe stress in heart muscle cells, promotes the release of free radicals, makes ischemia, and ultimately causes necrosis in the heart. In fact, high isoproterenol doses are used only to produce myocardial necrosis (10).

A new approach in regenerative medicine is the pretreatment of stem cells with antioxidants to reinforce the 
cells to increase their reparative property. Antioxidants suppress the formation of ROS and shift the balance toward antioxidants from pro-oxidants to protect myocytes (11). Treating cells by curcumin safeguards them against hydrogen peroxide exposure, which causes cell death and amplifies the differentiation of MSCs to osteoblasts (12). The pretreatment of MSCs with curcumin protects them against oxidative stress and enhances the efficacy of MSCs therapy (13). A recent study in a rat model of spinal injury revealed that co-therapy with curcumin and MSCs (60 $\mathrm{mg} / \mathrm{kg}$ weekly for four weeks) showed additional improvement in behavioral and locomotor recovery and a decrease in the inflammatory response (14). It has been demonstrated that curcumin can regulate the effect of BMSCs on collagen deposition and epidermal regeneration in cutaneous wound (15). BMSCs and curcumin treatment could improve the recovery of spinal injury synergically (16).

\section{Objectives}

In the present study, we aimed to examine the potency of curcumin-pretreated MSCs to improve MI by histopathological examination, serum biomarkers, and immunohistochemistry methods in rat model of MI. This investigation provides evidence that BMSCs treated with curcumin were more beneficial than cells without curcumin pretreatment in the treatment of myocardial ischemia.

\section{Methods}

\subsection{Animals}

We examined 36 adult male Wistar rats (17). The animals were fed ad libitum. They were kept at the Animal House of Urmia University of Medical Sciences in a standard controlled environment. The present study followed the guide for the care and use of laboratory animals of the Urmia University of Medical Sciences, Urmia, Iran, and received the ethical code of IR.umsu.Rec.1395.541.

\subsection{Induction of Experimental MI}

Isoproterenol hydrochloride $100 \mathrm{mg} / \mathrm{kg}$ (Sigma Chemical, USA) was injected to rats behind the neck subcutaneously on two consecutive days. The animals were euthanized 12 days after MI induction.

\subsection{Extraction of Stem Cells}

The marrow was extracted from the femurs of donor animals by the flushing method. The cells were centrifuged, and the supernatant was removed. The cell pellet was resuspended in a plastic flask containing DMEMLG (Gibco, Invitrogen) with 10\% FBS and 1\% antibioticantimycotic solution (Gibco, Invitrogen). When cells reached $80 \%$ confluence; then, adherent cells were detached via $0.05 \%$ trypsin (Invitrogen) for $2 \mathrm{~min}$. After the third passage, the cells reached the required dose and were seeded.

Curcumin (Cur; Sigma, USA) was dissolved in Dimethyl Sulphoxide (DMSO) (Sigma, USA) and added at a concentration of $10 \mu \mathrm{M} / \mathrm{L}$ into cell flasks for $48 \mathrm{~h}$ (18). Live-injected cells were detectable in the presence of red CM-DiI fluorescent stain (Invitrogen, USA).

\subsection{Animals}

We divided 36 Wistar rats into six groups of six rats. Two normal groups were injected with normal saline subcutaneously and four MI groups received Isoproterenol (ISO) $100 \mathrm{mg} / \mathrm{kg}$ in the same route. Group I (Normal 1) was treated with ethanol (curcumin solvent) orally for 12 days. Group II (Normal 2) was treated with infused DMEM via the tail vein. Group III (Iso/diseased control) was treated with ethanol orally for 12 days, and DMEM was infused via the tail vein. Group IV (Iso + curcumin) was treated with curcumin $80 \mathrm{mg} / \mathrm{kg}$ orally for 12 days. Group V (Iso + BMSCs) was treated with infused BMSCs $4 \times$ via the tail vein. Groups VI (Iso + Cur-BMSCs) was treated with infused BMSCs $4 \times$ (Cur pretreatment) via the tail vein. All animals were euthanized 12 days after treatment with curcumin or MSCs.

\subsection{Assessment of Myocardial Damage Markers}

Creatinine kinase-MB (CK-MB) and lactate dehydrogenase (LDH) were measured in serum samples by the standard kit (Pars Azemon Co., Iran) to determine the cardiac marker enzymes.

\subsection{Assessment of Antioxidant Enzymes}

The analysis of serum malondialdehyde (MDA) was performed by the spectrophotometry method to indicate lipid peroxidation. The superoxide dismutases (SOD) level was assessed by the photo-oxidation of o-dianisidine.

\subsection{Histopathological Examination}

Rats were euthanized and the apical parts of hearts were fixed in formalin and embedded in paraffin. Sections were stained with Hematoxylin and Eosin (H\&E) for the histological study and Masson's trichrome for detecting cardiac fibrosis. The features were graded, as follows: $1=$ low, $2=$ moderate $3=$ high, and $4=$ severe . 


\subsection{Measurement of MI Size}

To evaluate the infarct size, five consecutive slices were cut and then immersed in 5- triphenyl tetrazolium chloride $2 \%$ (TTC; Sigma, USA) for 20 min at room temperature. The slices were fixed in formalin $10 \%$ for one day and the color of infarcted areas changed to yellow-white. The infarcted myocardium in each slice was measured by Image J software (Version 1.46, National Institutes of Health, USA) as the ratio of ischemic area to the total slice.

\subsection{Immunohistochemistry Staining}

To determine the myocardial injury and death, tissues were deparaffinized and rehydrated to be assessed by a terminal deoxynucleotidyl transferase-mediated dUTP-biotin nick end labeling assay (TUNEL) apoptosis kit (Roche) according to the manufacturer's instruction. TUNEL-positive cells presented brown nuclei in the MI groups.

\subsection{Statistical Analysis}

Data were presented as mean \pm SEM. The one-way ANOVA was used to compare the groups. If the ANOVA indicated significant differences, the Tukey post hoc test was performed to compare the treatment and control groups in terms of the mean values. $\mathrm{P}<0.05$ showed significant differences between the groups.

\section{Results}

\subsection{Weight Parameters}

The effects of curcumin and curcumin-pretreated BMSCs on heart weight, body weight, and the ratio of heart weight to body weight are shown in Table 1 . All the parameters except for body weight were significantly higher in the ISO group than in controls. The heart weight and the ratio of heart weight to body weight were significantly lower in ISO + Cur, ISO + BMSCs, and ISO + Cur-BMSCs-treated groups than in the ISO group. The ratio of heart weight to body weight was significantly higher in the ISO + Cur and ISO + Cur-BMSCS-treated groups than in the BMSCs group.

\subsection{Effect of Curcumin and Curcumin-Pretreated BMSCs on Serum Enzyme Biomarkers}

The ischemic group showed a notable increase in the levels of CPK and LDH and the normal, ISO + Cur, ISO + BMSCs, and ISO + Cur-BMSCs-treated groups showed significantly lower levels of CPK and LDH than the ISO-treated group (Table 2). The levels of CPK and LDH were significantly lower in the ISO + Cur and, ISO + Cur-BMSCs treated groups than in the cell-treated group. Significantly higher levels of these biochemical markers were observed in the ISO group than in the control group $(\mathrm{P}<0.01)$.

\begin{tabular}{|c|c|c|c|}
\hline Treatment & Body wt. (g) & Heart wt. (g) & $\begin{array}{c}\text { Heart wt./Body wt. } \\
\text { Ratio }\end{array}$ \\
\hline Normal 1 & $284.6 \pm 6.62$ & $0.913 \pm 0.03$ & $3.19 \pm 0.06$ \\
\hline Normal 2 & $281.6 \pm 5.66$ & $0.916 \pm 0.02$ & $3.24 \pm 0.03$ \\
\hline ISO & $253 \pm 3.24$ & $1.033 \pm 0.02^{\mathrm{a}}$ & $4.08 \pm 0.07^{\mathrm{b}}$ \\
\hline ISO + Cur & $278.83 \pm 3.57$ & $0.968 \pm 0.01^{\mathrm{b}}$ & $3.46 \pm 0.02^{c, d}$ \\
\hline ISO + BMSCs & $271.17 \pm 6.73$ & $1.033 \pm 0.01$ & $3.80 \pm 0.07^{c}$ \\
\hline ISO + Cur-BMSCs & $283.82 \pm 5.29$ & $1.005 \pm 0.02$ & $3.54 \pm 0.05^{c, d}$ \\
\hline \multicolumn{4}{|c|}{$\begin{array}{l}\text { Abbreviation: BMSCs, Bone Marrow Mesenchymal Stem Cells. } \\
\text { aa Values are expressed as mean } \pm \text { SEM. } \\
{ }^{b} \text { P values }<0.05 \text { compared to normal. } \\
{ }^{c} \text { P values }<0.05 \text { compared to ISO. } \\
{ }^{d} \text { P values }<0.05 \text { compared to ISO + BMSCs. }\end{array}$} \\
\hline
\end{tabular}

Table 2. Effect of Curcumin and Curcumin-Pretreated BMSCs on CK and LDH Enzyme Activities in Treated Rats ${ }^{\mathrm{a}}$

\begin{tabular}{|c|c|c|}
\hline Treatment & LDH & CPK \\
\hline Normal 1 & $243 \pm 4.3$ & $233.33 \pm 6.6$ \\
\hline Normal 2 & $251.50 \pm 4.6$ & $222.50 \pm 6.3$ \\
\hline ISO & $387.75 \pm 13.1^{\mathrm{b}}$ & $395.25 \pm 2.0^{\mathrm{b}}$ \\
\hline ISO + Cur & $268.50 \pm 6.1^{c, d}$ & $260.25 \pm 4.7^{\mathrm{c}, \mathrm{d}}$ \\
\hline ISO + BMSCS & $329.67 \pm 15.0^{c}$ & $317.67 \pm 17.6^{c}$ \\
\hline ISO +Cur-BMSCs & $287.67 \pm 7.8^{\mathrm{c}, \mathrm{d}}$ & $258.75 \pm 9.0^{\mathrm{c}, \mathrm{d}}$ \\
\hline
\end{tabular}

\subsection{Effect of Curcumin and Curcumin-Pretreated BMSCs on Serum Oxidant Status}

The effects of curcumin on lipid peroxidation and antioxidant enzymes are shown in Table 3. The activities of serum MDA and SOD were significantly higher $(\mathrm{P}<0.001)$ in the ISO group than in normal rats. The activities of these enzymes were significantly lower $(P<0.001)$ in Cur and Cur-BMSCs groups than in BMSCs rats.

\subsection{Histopathological Examination of Cardiac Tissue}

The histological observations rated based on the intensity of damage are shown in Figure 1. There was no evidence of necrosis, edema, and inflammation in the normal group. Ischemic hearts showed myocardial necrosis with cell infiltration, edema, and increased connective tissue between cardiac muscle fibers whereas rats treated with Cur, BMSCs, and Cur-BMSCs after MI induction demonstrated less myonecrosis and edema with minimal inflammation. The observed protection was more with Cur and Cur-BMSCs than with BMSCs against myocardial necrosis. Effects of curcumin and Curcumin-pretreated BMSCs on cardiomyocyte fibrosis. 
A
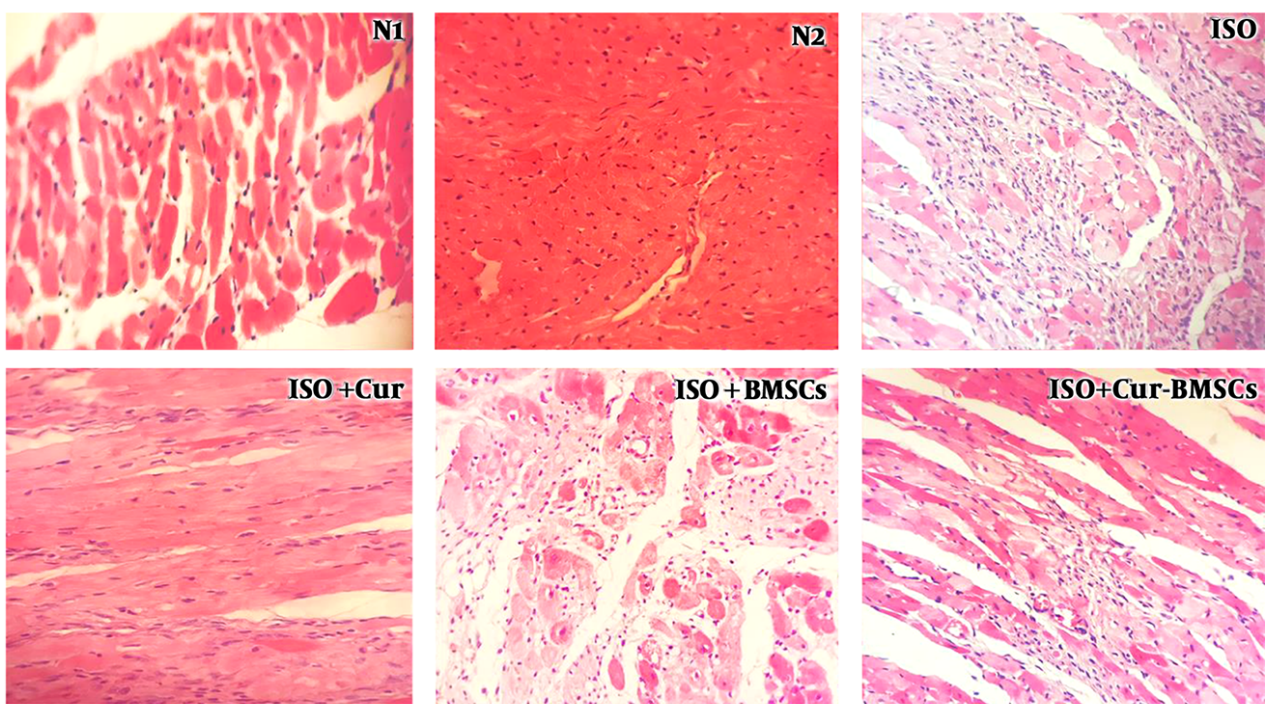

B

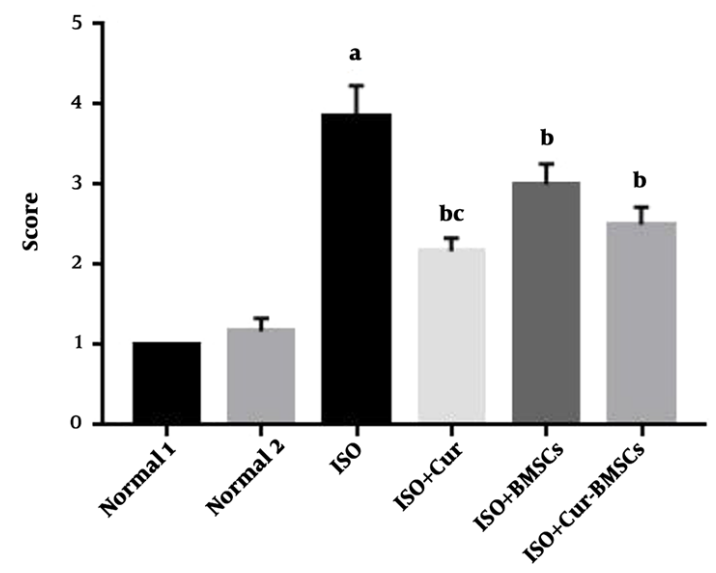

Figure 1. A, photomicrographs of the sections of rat's cardiac apexes. Heart tissue of rat treated with isoproterenol(ISO) shows intensive cardiomyocyte necrosis and increased edematous intramuscular space. Acute treatment with curcumin (Cur), bone marrow stem cells (BMSCs), and Cur-pretreated BMSCs (Cur-BMSCs) demonstrated a marked improvement. (H\&E staining at 400 magnification). B, grading of histopathological changes in rat's cardiac apex tissue. Grades 1, 2, 3, and 4 show low, moderate, high, and intensive pathological changes, respectively. ISO, isoproterenol; Cur, curcumin; BMSCs, bone marrow stem cells; Cur-BMSCs, Cur-pretreated BMSCs. Values are mean \pm SEM (n =6). P values $<0.001$ compared to (a) normal, (b) ISO, and (c) ISO + BMSCs.

The arrangement of cardiac muscle fibers was regular with obvious boundary in the normal group after Masson Trichrome staining (Figure 2). No apparent degeneration or fibrosis was observed in the normal group. A severe grade of fibrosis was recognized as blue-colored areas in the microscopic slides of ischemic hearts. Cur and BMSCs and Cur-BMSCs decreased inflammatory reactions and cardiac fibrosis. It was noticed that Cur, BMSCs, and CurBMSCs could reduce isoproterenol-induced fibrosis.
4.5. Effects of Curcumin and Curcumin-Pretreated BMSCs on Infarct Size

The TTC staining of heart tissues in ISO + Cur, ISO+BMSCs, and ISO + Cur-BMSCs groups showed the reduced infarct size following MI induction (Figure 3). Analysis of measurements revealed that the infarct size was remarkably smaller in ISO + Cur, ISO+BMSCs, and ISO + Cur-BMSCs than in the ISO group. The infarct size was also significantly smaller in the ISO + Cur and ISO + Cur-BMSCs groups than in the BMSCs group. 

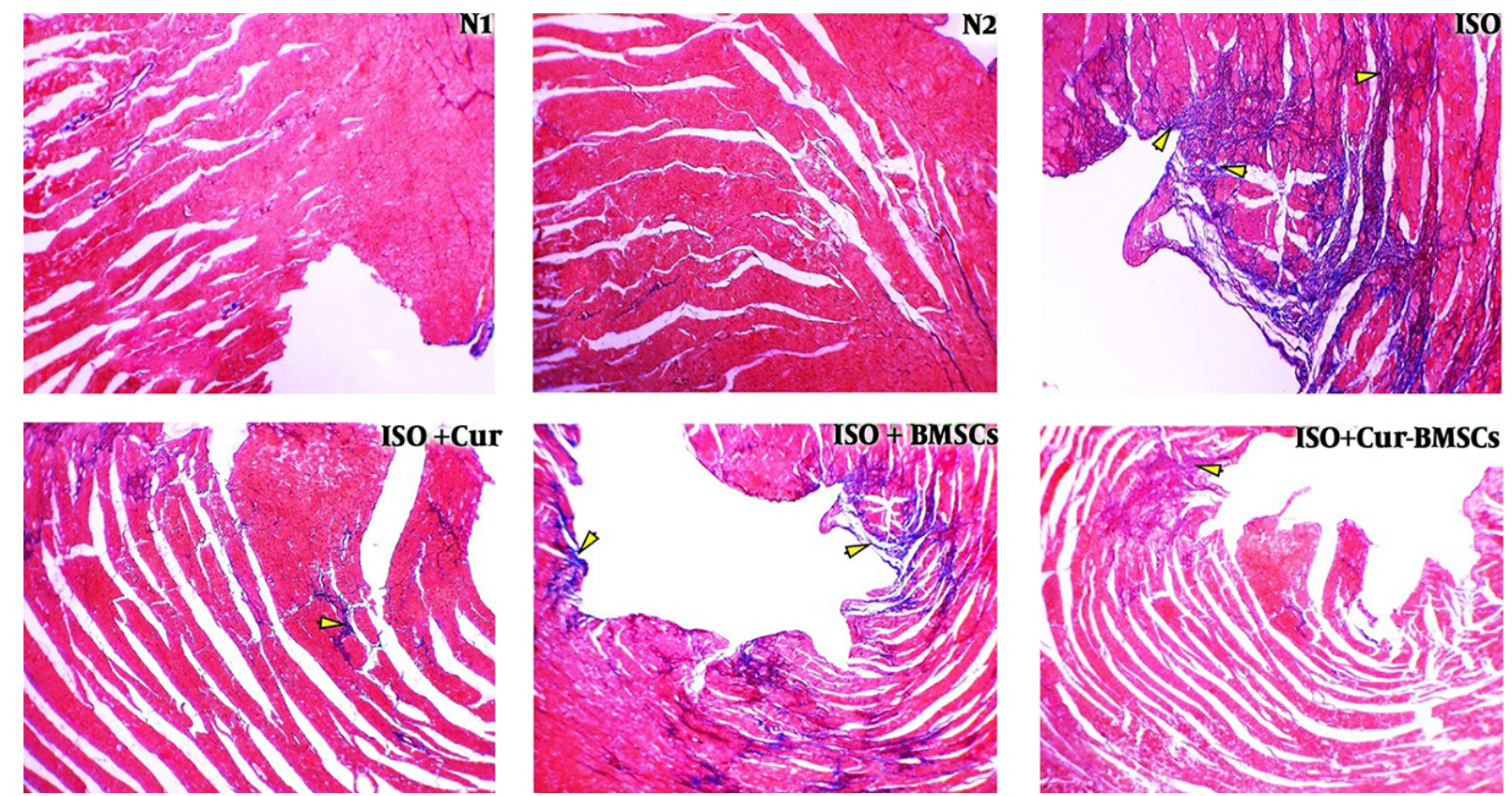

Figure 2. Photomicrographs of Masson Trichrome stained sections of rat's cardiac apexes. Fibrotic tissues were stained blue and cardiomyocytes were stained red. The myocardial fibers were arranged regularly with clear striations in the normal control groups. No apparent degeneration or fibrosis was observed in the normal control group. Isoproterenol-treated hearts showed a severe grade of fibrosis recognized as blue-colored parts $63 \%(\mathrm{P}<0.001)$. It was observed that treatment with Cur $36 \%$ and BMSCs $52 \%$ and Cur-BMSCs 38\% the fibrosis ( $\mathrm{P}<0.01$ ). Collagen deposition was quantified by an automated Image analyzer and expressed as the percentage of tissue area. The data are expressed as means \pm SEM ( $\mathrm{n}=6$ for each group). ISO: isoproterenol; Cur, curcumin; BMSCs, bone marrow stem cells; Cur-BMSCs, Cur-pretreated BMSCs. (400 magnification).

Table 3. Effect of Curcumin and Curcumin-Pretreated BMSCs on Oxidative Status in Treated Rats ${ }^{\mathrm{a}}$

\begin{tabular}{lcc}
\hline Treatment & SOD $(\mathbf{u} / \mathbf{m L})$ & MDA $(\mathbf{n m o l} / \mathbf{m L})$ \\
\hline Normal 1 & $54.0 \pm 1.00$ & $6.31 \pm .13$ \\
\hline Normal 2 & $56.3 \pm 0.95$ & $6.30 \pm .14$ \\
\hline ISO & $98.5 \pm 0.99^{\mathrm{b}}$ & $14.30 \pm .12^{\mathrm{b}}$ \\
\hline ISO + Cur & $69.7 \pm 4.38^{\mathrm{c}, \mathrm{d}}$ & $7.81 \pm .35^{\mathrm{c}, \mathrm{d}}$ \\
\hline ISO + BMSCs & $82.2 \pm 1.60^{\mathrm{c}}$ & $10.43 \pm .38^{\mathrm{c}}$ \\
\hline ISO + Cur- BMSCs & $67.4 \pm 1.28^{\mathrm{c}, \mathrm{d}}$ & $7.68 \pm .37^{\mathrm{c}, \mathrm{d}}$ \\
\hline
\end{tabular}

${ }^{\mathrm{a}}$ Values are expressed as mean \pm SEM.

${ }^{\mathrm{b}}$ P values $<0.001$ compared to normal.

${ }^{c} \mathrm{P}$ values $<0.001$ compared to ISO

${ }^{\mathrm{d}} \mathrm{P}$ values $<0.001$ compared to ISO + BMSCs

\subsection{Tunnel Positive Myocardium}

Cardiac muscles with tan nuclei were considered tunnel-positive cells. The nuclei of myocardial cells of rats in the normal group (Figure 4) were blue with almost no evidence of apoptosis. However, 12 days after MI induction, the cells surrounding the damaged area were mostly tunnel-positive in the ISO group, with a statistically significant difference from the normal group. The number of apoptotic cells was significantly lower in three treatment groups than in the ISO group (Table 4) and in the Cur and

\begin{tabular}{|c|c|}
\hline Treatment & Apoptosis Index (\%) \\
\hline Normal 1 & $6.51 \pm 0.17$ \\
\hline Normal 2 & $7.30 \pm 0.18$ \\
\hline ISO & $48.57 \pm 0.92^{\mathrm{b}}$ \\
\hline ISO + Cur & $28.81 \pm 0.55^{c, d}$ \\
\hline ISO + BMSCs & $37.43 \pm 0.78^{\mathrm{C}}$ \\
\hline ISO + Cur-BMSCs & $30.68 \pm 0.67^{c, d}$ \\
\hline $\begin{array}{l}{ }^{\mathrm{a}} \text { Values are express } \\
{ }^{\mathrm{b}} \mathrm{P} \text { values }<0.001 \mathrm{c} \\
{ }^{\mathrm{c}} \mathrm{P} \text { values }<0.001 \mathrm{c} \\
{ }^{\mathrm{d}} \text { P values }<0.001 \mathrm{c}\end{array}$ & \\
\hline
\end{tabular}

Cur-BMSCs groups than in the BMSCs group. The difference was not significant between the Cur and Cur-BMSCs groups $(\mathrm{P}<0.001)$.

\section{Discussion}

Myocardial infarction is the main cause of mortality worldwide. MSCs are the best candidates for the regeneration of myocardium after the decreased number of cardiomyocytes in the ischemic heart. Many reports have 
$\mathbf{A}$
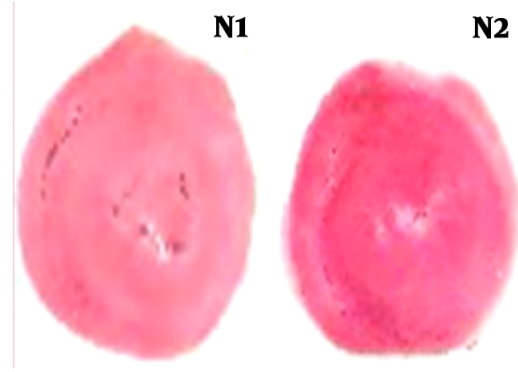

N2

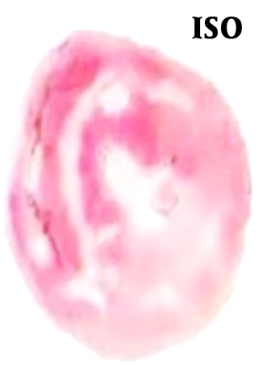

ISO

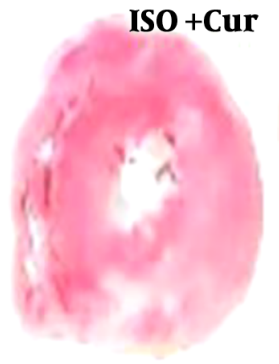

ISO + BMSCs

ISO+Cur-BMSCs

\section{B}

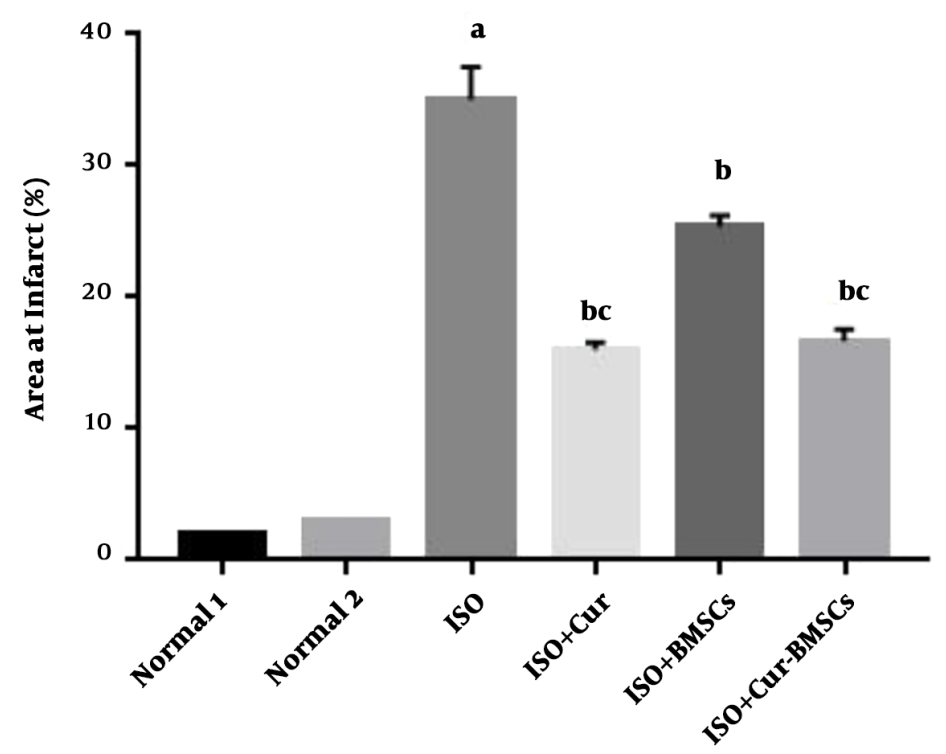

Figure 3. Effect of Cur, BMSCs, and Cur-pretreated BMSCs on infarct size. Representative TTC staining images by quantitative analysis at 12 days after myocardial infarction showed the decreased heart infarct size. The values are mean \pm SEM; P values $<0.05$ compared to (a) normal, (b) ISO, and (c) ISO+BMSCs.

demonstrated that MSCs with their ability to differentiate into cardiomyocyte-shaped cells can improve heart function and significantly repair fibrous tissue (19). Intravenous injection of MSCs is the most available method, while the migration of infused cells to the site of the disease has been shown in myocardial ischemic models (20). This route is safe with a minimum invasion that requires no expensive lab instruments with MSCs successful homing (21). Curcumin is a herbal food additive with antioxidant and anti-inflammatory activities. The cardioprotective influence of curcumin has been documented in patients and animal models (22).

We found only one report of reduced apoptosis and increased angiogenesis by pretreating adipose-derived stem cells with curcumin (11). Our study disclosed that curcumin alone and its combination with MSCs exerted significant beneficial effects against MI. Our results also demonstrated that isoproterenol $100 \mathrm{mg} / \mathrm{kg}$ for two days could increase the heart weight, inflammation, and interfiber space, which were characteristic to typical MI. Similar results were observed in other reports after the induction of MI (17). Oral gavage of curcumin $80 \mathrm{mg} / \mathrm{kg}$ for 12 days and curcumin-pretreated MSCs could regenerate myocardial injury, edematous, and major fibrosis in treatment groups. Curcumin compensates for free radical injuries and protects heart fibers against oxidative stress (23). Stem cells are exposed to some stressors during their lives and lose their survival when experiencing the aging process. An im- 

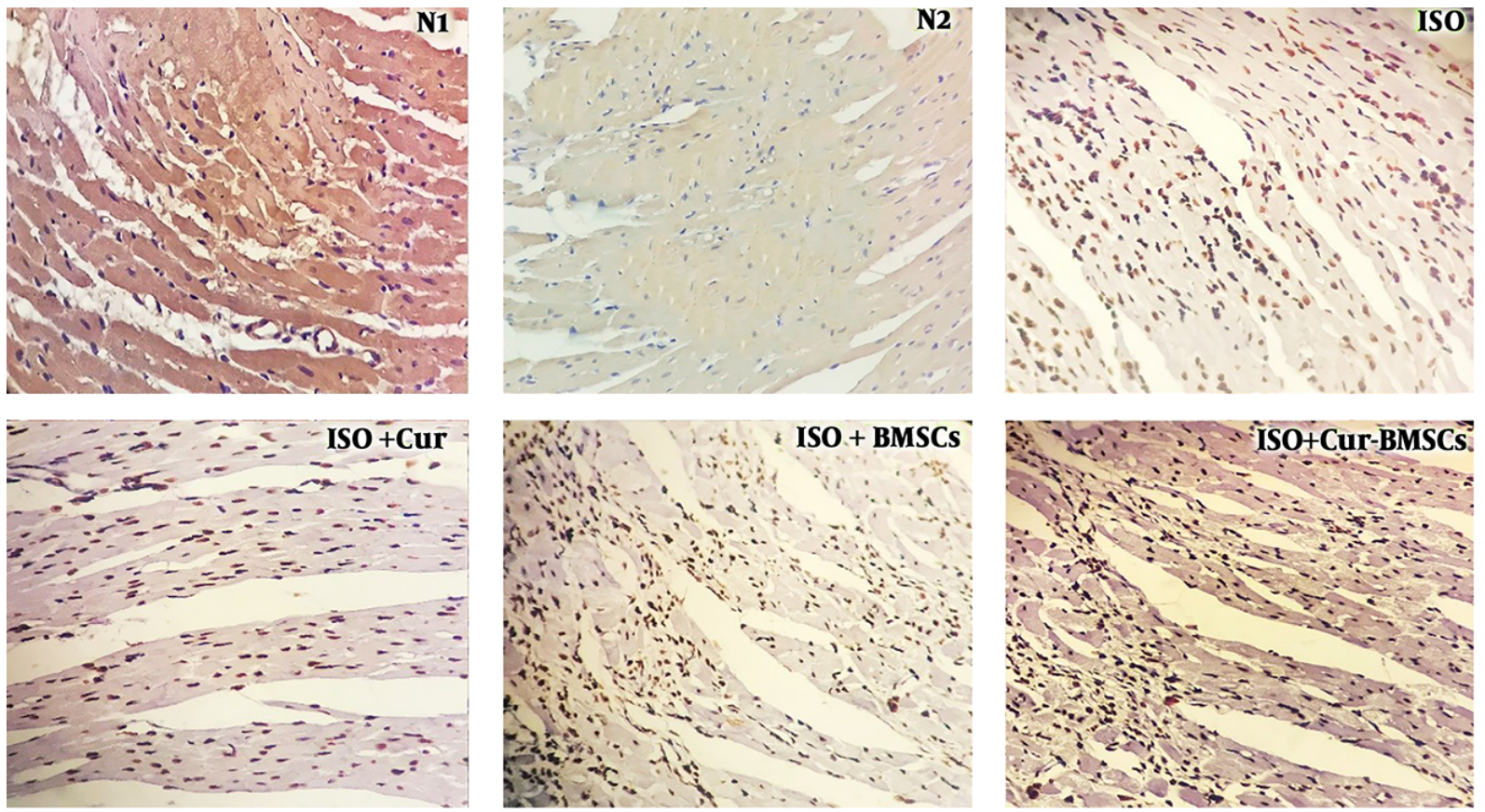

Figure 4. Photomicrographs of TUNEL stained sections of rat's cardiac apexes. The apoptotic cells were predominant in the ISO group compared to the normal group. The number of apoptotic cells was lower in the curcumin and BMSCs groups than in the ISO group. The Cur and Cur-BMSCs groups were significantly different from the BMSCs group. The data are expressed as means \pm SEM ( $n=6$ for each group). ISO, isoproterenol; Cur, curcumin; BMSCs, bone marrow stem cells; Cur-BMSCs, Cur-pretreated BMSCs (400 magnification).

portant indicator of oxidative stress is the increased ROS generation that, in turn, leads to the impairment of therapeutic potency of stem cells (24). It has been reported that curcumin protects the survival and differentiation of human adipose-derived stem cells against oxidative damage by increasing Wnt/ $\beta$-Catemin signaling (12).

The intravenous injection of pretreated MSCs and oral administration of curcumin strongly reduced the infarct size. Our result is in line with a recently published paper that showed a significant reduction in the infarct size following the administration of curcumin $30 \mathrm{mg} / \mathrm{kg}$ in rats (25). In addition, our study confirms an earlier report of the reduced myocardial infarct size following the intravenous infusion of BMSCs (21). This reduction is related to the decreased level of some markers of myocardial injury such as LDH and CK-MB as the typical biomarkers of mortality (25).

The significant increase in lactate dehydrogenase (LDH) and Creatine Kinase (CK-MB) demonstrated the presence of MI in the ISO-treated group. These results accord with previous studies that used the induction of MI with isoproterenol (26). The myocardium contains cardiac enzymes such as CK-MB and LDH. After the induction of myocardial ischemia and increased oxygen demand, the cell membrane is damaged, and cardiac enzymes leakage into the blood. CK-MB is a remarkable cardiac enzyme, and its increase is a valuable diagnostic marker for MI (27).

It is believed that after intravenous injection of MSCs to damaged heart, only a small percentage of injected cells are differentiated to cardiomyocytes. Many trophic factors, including proteins and lipids, as well as anti-inflammatory factors, can secrete from MSCs and their paracrine activities may be responsible for the regeneration of damaged tissue (28). An increase in oxidative stress markers following MI has been reported by many researchers (29) and this may damage lipids and proteins of cardiomyocytes. It has been reported that curcumin has a regulatory effect on cardiac parameters, myocardial injury, and mitochondrial glutathione, due to its antioxidants effects (30). Pretreatment of MSCs with curcumin resulted in a significant reduction in apoptosis in the myocardium. A recent study revealed that treated rats with curcumin $150 \mathrm{mg} / \mathrm{kg}$ showed reduced apoptotic and inflammatory cells and decreased fibrous tissue in the infarcted area. It has been indicated that curcumin significantly increases BCL-2 that, in turn, suppresses the initiation of apoptosis (31). The permanent decrease in the number of heart myocytes in the ISO group was apparent and regenerative treatment through cell therapy was effective for MI treatment (32).

It has been shown that curcumin attenuates MI- 
induced oxidative stress, but all these changes were dosedependent and were pronounced at higher doses (25). Evidence supports the ability of engrafted MSCs in the treatment of ischemic heart disease, regulation of cell survival, growth, proliferation, differentiation, and migration to the damaged area of the heart. The possible mechanism is related to increased glucose metabolism in the myocardium and protein synthesis through the secretion of cytokines by MSCs (33). Both MSCs and curcumin have shown beneficial effects on the treatment of MI and improve cardiac function but our study, for the first time, provides evidence that the pretreatment of BMSCs has the reparative influence on the heart morphology and function.

\section{Footnotes}

Conflict of Interests: The authors declare that they have no conflict of interest.

\section{Ethical Approval: IR.umsu.Rec.1395.541.}

Funding/Support: The Research Deputy of Urmia University of Medical Sciences, Urmia, Iran, funded the study.

\section{References}

1. Goyal SN, Sharma C, Mahajan UB, Patil CR, Agrawal YO, Kumari $S$, et al. Protective effects of cardamom in isoproterenol-induced myocardial infarction in rats. Int J Mol Sci. 2015;16(11):27457-69. doi: 10.3390/ijms161126040. [PubMed: 26593900]. [PubMed Central: PMC4661898].

2. Patel P, Parikh M, Shah H, Gandhi T. Inhibition of RhoA/Rho kinase by ibuprofen exerts cardioprotective effect on isoproterenol induced myocardial infarction in rats. Eur J Pharmacol. 2016;791:91-8. doi: 10.1016/j.ejphar.2016.08.015. [PubMed: 27565218].

3. Lobo Filho HG, Ferreira NL, Sousa RB, Carvalho ER, Lobo PL, Lobo Filho JG. Experimental model of myocardial infarction induced by isoproterenol in rats. Rev Bras Cir Cardiovasc. 2011;26(3):469-76. doi: 10.5935/1678-9741.20110024. [PubMed: 22086586].

4. Liu K, Chen H, You QS, Ye Q, Wang F, Wang S, et al. Curcumin attenuates myocardial ischemia-reperfusion injury. Oncotarget. 2017;8(67):112051-9. doi: 10.18632/oncotarget.23002. [PubMed: 29340110]. [PubMed Central: PMC5762378].

5. Bian S, Zhang L, Duan L, Wang X, Min Y, Yu H. Extracellular vesicles derived from human bone marrow mesenchymal stem cells promote angiogenesis in a rat myocardial infarction model. $J \mathrm{Mol}$ Med (Berl). 2014;92(4):387-97. doi: 10.1007/s00109-013-1110-5. [PubMed: 24337504].

6. Cai B, Tan X, Zhang Y, Li X, Wang X, Zhu J, et al. Mesenchymal stem cells and cardiomyocytes interplay to prevent myocardial hypertrophy. Stem Cells Transl Med. 2015;4(12):1425-35. doi: 10.5966/sctm.20150032. [PubMed: 26586774]. [PubMed Central: PMC4675503].

7. Zhang GW, Gu TX, Guan XY, Sun XJ, Qi X, Li XY, et al. bFGF binding cardiac extracellular matrix promotes the repair potential of bone marrow mesenchymal stem cells in a rabbit model for acute myocardial infarction. Biomed Mater. 2015;10(6):65018. doi: 10.1088/17486041/10/6/065018. [PubMed: 26657457].

8. Hua P, Wang YY, Liu LB, Liu JL, Liu JY, Yang YQ, et al. In vivo magnetic resonance imaging tracking of transplanted superparamagnetic iron oxide-labeled bone marrow mesenchymal stem cells in rats with myocardial infarction. Mol Med Rep. 2015;11(1):113-20. doi: 10.3892/mmr.2014.2649. [PubMed: 25323652]. [PubMed Central: PMC4237077].

9. Chi NH, Yang MC, Chung TW, Chen JY, Chou NK, Wang SS. Cardiac repair achieved by bone marrow mesenchymal stem cells/silk fibroin/hyaluronic acid patches in a rat of myocardial infarction model. Biomaterials. 2012;33(22):5541-51. doi 10.1016/j.biomaterials.2012.04.030. [PubMed: 22575829].

10. Rathore N, John S, Kale M, Bhatnagar D. Lipid peroxidation and antioxidant enzymes in isoproterenol induced oxidative stress in rat tissues. Pharmacol Res. 1998;38(4):297-303. doi: 10.1006/phrs.1998.0365. [PubMed: 9774493].

11. Liu J, Zhu P, Song P, Xiong W, Chen H, Peng W, et al. Pretreatment of adipose derived stem cells with curcumin facilitates myocardial recovery via antiapoptosis and angiogenesis. Stem Cells Int. 2015;2015:638153. doi: 10.1155/2015/638153. [PubMed: 26074974]. [PubMed Central: PMC4436501].

12. Wang N, Wang F, Gao Y, Yin P, Pan C, Liu W, et al. Curcumin protects human adipose-derived mesenchymal stem cells against oxidative stress-induced inhibition of osteogenesis. J Pharmacol Sci. 2016;132(3):192-200. doi: 10.1016/j.jphs.2016.10.005. [PubMed: 27840063].

13. Ao XX, Huang H. Curcumin protects mesenchymal stem cells against oxidative stress-induced apoptosis via Akt/mTOR/p70S6K pathway. Int J Clin Exp Pathol. 2017;10(6):6655-64.

14. Ruzicka J, Urdzikova LM, Kloudova A, Amin AG, Vallova J, Kubinova S, et al. Anti-inflammatory compound curcumin and mesenchymal stem cells in the treatment of spinal cord injury in rats. Acta Neurobiol Exp (Wars). 2018;78(4):358-74. [PubMed: 30624435].

15. Yang Z, He C, He J, Chu J, Liu H, Deng X. Curcumin-mediated bone marrow mesenchymal stem cell sheets create a favorable immune microenvironment for adult full-thickness cutaneous wound healing. Stem Cell Res Ther. 2018;9(1):21. doi:10.1186/s13287-018-0768-6. [PubMed 29386050]. [PubMed Central: PMC5793416].

16. Ormond DR, Shannon C, Oppenheim J, Zeman R, Das K, Murali R, et al. Stem cell therapy and curcumin synergistically enhance recovery from spinal cord injury. PLoS One. 2014;9(2). e88916. doi 10.1371/journal.pone.0088916. [PubMed: 24558450]. [PubMed Central: PMC3928327].

17. Soraya H, Khorrami A, Garjani A, Maleki-Dizaji N, Garjani A. Acute treatment with metformin improves cardiac function following isoproterenol induced myocardial infarction in rats. Pharmacol Rep. 2012;64(6):1476-84. [PubMed: 23406758].

18. Attari F, Zahmatkesh M, Aligholi H, Mehr SE, Sharifzadeh M, Gorji A, et al. Curcumin as a double-edged sword for stem cells: Dose time and cell type-specific responses to curcumin. Daru. 2015;23:33. doi: 10.1186/s40199-015-0115-8. [PubMed: 26063234]. [PubMed Central: PMC4466857].

19. Nagaya N, Kangawa K, Itoh T, Iwase T, Murakami S, Miyahara Y et al. Transplantation of mesenchymal stem cells improves cardiac function in a rat model of dilated cardiomyopathy. Circulation. 2005;112(8):1128-35. doi: 10.1161/CIRCULATIONAHA.104.500447. [PubMed: 16103243].

20. Barbash IM, Chouraqui P, Baron J, Feinberg MS, Etzion S, Tessone A, et al. Systemic delivery of bone marrow-derived mesenchymal stem cells to the infarcted myocardium: Feasibility, cell migration, and body distribution. Circulation. 2003;108(7):863-8. doi 10.1161/01.CIR.0000084828.50310.6A. [PubMed:12900340].

21. Mykhaylichenko VY, Kubyshkin AV, Samarin SA, Fomochkina II Anisimova LV. Experimental induction of reparative morphogenesis and adaptive reserves in the ischemic myocardium using multipotent mesenchymal bone marrow-derived stem cells. Pathophysiology. 2016;23(2):95-104. doi: 10.1016/j.pathophys.2016.04.002. [PubMed 27102896].

22. Jiang S, Han J, Li T, Xin Z, Ma Z, Di W, et al. Curcumin as a potential protective compound against cardiac diseases. Pharmacol Res. 2017;119:373-83. doi: 10.1016/j.phrs.2017.03.001. [PubMed: 28274852]. 
23. Chakraborty M, Bhattacharjee A, Kamath JV. Cardioprotective effect of curcumin and piperine combination against cyclophosphamideinduced cardiotoxicity. Indian J Pharmacol. 2017;49(1):65-70. doi: 10.4103/0253-7613.201015. [PubMed: 28458425]. [PubMed Central: PMC5351241].

24. Li L, Guo Y, Zhai H, Yin Y, Zhang J, Chen H, et al. Aging increases the susceptivity of MSCs to reactive oxygen species and impairs their therapeutic potency for myocardial infarction. PLoS One. 2014;9(11). e111850. doi: 10.1371/journal.pone.0111850. [PubMed: 25393016]. [PubMed Central: PMC4230939].

25. Liu H, Wang C, Qiao Z, Xu Y. Protective effect of curcumin against myocardium injury in ischemia reperfusion rats. Pharm Biol. 2017;55(1):1144-8. doi: 10.1080/13880209.2016.1214741. [PubMed: 28224816]. [PubMed Central: PMC6130472].

26. Wang SB, Tian S, Yang F, Yang HG, Yang XY, Du GH. Cardioprotective effect of salvianolic acid A on isoproterenol-induced myocardial infarction in rats. Eur J Pharmacol. 2009;615(1-3):125-32. doi 10.1016/j.ejphar.2009.04.061. [PubMed: 19445921].

27. Loh HK, Sahoo KC, Kishore K, Ray R, Nag TC, Kumari S, et al. Effects of thalidomide on isoprenaline-induced acute myocardial injury: A haemodynamic, histopathological and ultrastructural study. Basic Clin Pharmacol Toxicol. 2007;100(4):233-9. doi: 10.1111/j.17427843.2007.00022.x. [PubMed: 17371527].

28. Baglio SR, Pegtel DM, Baldini N. Mesenchymal stem cell secreted vesicles provide novel opportunities in (stem) cell-free therapy. Front Physiol. 2012;3:359. doi: 10.3389/fphys.2012.00359. [PubMed:
22973239]. [PubMed Central: PMC3434369].

29. Lefer DJ, Granger DN. Oxidative stress and cardiac disease. Am J Med. 2000;109(4):315-23. doi: 10.1016/s0002-9343(00)00467-8. [PubMed: 10996583].

30. Ilyas EI, Nur BM, Laksono SP, Bahtiar A, Estuningtyas A, Vitasyana $\mathrm{C}$, et al. Effects of curcumin on parameters of myocardial oxidative stress and of mitochondrial glutathione turnover in reoxygenation after 60 minutes of hypoxia in isolated perfused working guinea pig hearts. Adv Pharmacol Sci. 2016;2016:6173648. doi: 10.1155/2016/6173648. [PubMed: 26904113]. [PubMed Central: PMC4745620].

31. Lv FH, Yin HL, He YQ, Wu HM, Kong J, Chai XY, et al. Effects of curcumin on the apoptosis of cardiomyocytes and the expression of NFkappaB, PPAR-gamma and Bcl-2 in rats with myocardial infarction injury. Exp Ther Med. 2016;12(6):3877-84. doi: 10.3892/etm.2016.3858. [PubMed: 28105120]. [PubMed Central: PMC5228430].

32. Williams AR, Hare JM. Mesenchymal stem cells: Biology, pathophysiology, translational findings, and therapeutic implications for cardiac disease. Circ Res. 2011;109(8):923-40. doi: 10.1161/CIRCRESAHA.111.243147. [PubMed: 21960725]. [PubMed Central: PMC3604746].

33. Cai M, Shen R, Song L, Lu M, Wang J, Zhao S, et al. Bone marrow mesenchymal stem cells (BM-MSCs) improve heart function in swine myocardial infarction model through paracrine effects. Sci Rep. 2016;6:28250. doi: 10.1038/srep28250. [PubMed: 27321050]. [PubMed Central: PMC4913323]. 